\title{
Extremes and Products of Multivariate AC-Product Risks
}

\author{
Yang Yang $^{1}$ \& Enkelejd Hashorva ${ }^{2}$
}

January 16, 2013

\begin{abstract}
With motivation from Tang et al. (2011), in this paper we consider a tractable multivariate risk structure which includes the Sarmanov dependence structure as a special case. We derive several asymptotic results for both the sum and the product of such risk and then present three applications related to actuarial mathematics.

Keywords: AC-product distribution; Sarmanov distribution; Random deflators; Risk aggregation; Ruin probability;
\end{abstract}

2000 Mathematics Subject Classification: 60G70; 62E20; 62H20

\section{Introduction}

Many modern actuarial tasks such as quantification of large risks and aggregated risk, estimation of ruin probabilities in the presence of financial risks, or reinsurance pricing accounting for both claims and expenses strongly rely on the use of multivariate extreme value theory. Typically, the adequacy of the probabilistic models employed by the actuaries is determined by their flexibility to allow for the dependence among risks. Most of classical insurance models assume independence of risks, a phenomenon which is rarely observed in practical actuarial tasks. The role of the dependence among risks is crucial, especially when modelling the impact of large risks. Dependence modeling and in particular that of large risks has been the topic of several contributions such as Goovaerts et al. (2005), Denuit et al. (2006), Li et al. (2010), Asimit et al. (2011), Chen (2011), Haug et al. (2011), Manner and Segers (2011), Tang et al. (2011), Chen and Yuen (2009,2012) among many others.

Asimit et al. (2011) successfully demonstrates the role of asymptotic dependence and asymptotic independence in actuarial modelling. As shown therein, multivariate risks which exhibit asymptotic dependence imply in general different results compared to multivariate risks which have asymptotic independent components. Tractable multivariate distributions like the Fairlie-Gumbel-Morgenstern (FGM) ones exhibit asymptotic independence. In various risk models employed by actuaries two related tasks are the asymptotic analysis of aggregated risk, and the asymptotic quantification of the effect random scaling (or deflation) of risks. Since the empirical data always support the fact that risks are stochastically dependent, aggregation of dependent risks has become recently a key topic for insurance, finance, and risk management. Recent results of Mitra and Resnick (2009) and Asimit et al. (2011) pave the way for the analysis of the impact of a single large risk to the aggregated risk. In a mathematical framework, if $X_{0}, \ldots, X_{n}$ are non-negative random variables (rv's) with distribution functions (df's) $F_{0}, \ldots, F_{n}$, then the aggregated risk is $S=\sum_{i=0}^{n} X_{i}$. In order to avoid triviality, we assume that the risks are all non-degenerate at zero. Large values of $S$ mean large financial risks for the company, and therefore the actuarial interest focusses mainly on the quantification of the probability of such large values, i.e., $\mathbb{P}(S>u)$ where the level $u$ reaches some extreme point.

In another context, $X_{0}$ can be considered as the base risk, whereas $X_{1}, \ldots, X_{n}$ as random deflators/inflators.

\footnotetext{
${ }^{1}$ School of Mathematics and Statistics, Nanjing Audit University, Nanjing 210029, China, School of Economics and Management, Southeast University, Nanjing 210096, China

${ }^{2}$ University of Lausanne, UNIL-Dorigny 1015 Lausanne, Switzerland
} 
Of actuarial interest is the asymptotic tail behaviour of the ultimate deflated risk $(u \rightarrow \infty)$

$$
\mathbb{P}(Z>u), \quad \text { with } Z=X_{0} \prod_{i=1}^{n} X_{i}
$$

For independent risks recent results in this direction are derived in Hashorva at al. (2010).

The main goal of this paper is to introduce a tractable class of dependent risks which allows for explicit calculation of various actuarial quantities of interest. The motivation for introducing such a class of risks comes from the simple structure of multivariate FGM df's. By definition, a $(n+1)$-dimensional random vector $\boldsymbol{X}=\left(X_{0}, \ldots, X_{n}\right)$ has a multivariate FGM df $Q$ with marginal df's $F_{0}, \ldots, F_{n}$ if

$$
Q\left(x_{0}, \ldots, x_{n}\right)=\prod_{i=0}^{n} F_{i}(x)\left[1+\sum_{0 \leq i<j \leq n} \theta_{i j} \overline{F_{i}}\left(x_{i}\right) \overline{F_{j}}\left(x_{j}\right)\right], \quad x_{i} \in\left[0, \hat{x}_{i}\right], 0 \leq i \leq n,
$$

where $\overline{F_{i}}:=1-F_{i}$, and $\theta_{i j}$ 's are some real constants which satisfy certain restrictions so that $Q$ is a df. Here $\hat{x}_{i}:=\sup \left\{x \in \mathbb{R}: F_{i}(x)<1\right\}$ stands for the upper endpoint of the marginal df $F_{i}$.

Throughout the paper we assume that the risks are non-negative, thus $F_{i}$ has support on $[0, \infty)$.

The tractability of $\boldsymbol{X}$ with df $Q$ given by (1.2) relates to the fact that $Q$ is obtained by the product distribution $Q^{*}=\prod_{i=0}^{n} F_{i}$ (in fact $\left.Q^{*}\left(x_{0}, \ldots, x_{n}\right)=\prod_{i=0}^{n} F_{i}\left(x_{i}\right)\right)$. By a closer inspection, it follows that

$$
Q\left(x_{0}, \ldots, x_{n}\right)=\int_{0}^{x_{0}} \cdots \int_{0}^{x_{n}}\left[1+\sum_{0 \leq i<j \leq n} \theta_{i j}\left(1-2 F_{i}\left(s_{i}\right)\right)\left(1-2 F_{j}\left(s_{j}\right)\right)\right] Q^{*}\left(\mathrm{~d} s_{0}, \ldots, \mathrm{d} s_{n}\right)
$$

holds for any $x_{i} \in\left[0, \hat{x}_{i}\right], 0 \leq i \leq n$. The larger class of multivariate Sarmanov distributions is introduced by substituting above $1-2 F_{i}$ by some kernel $\phi_{i}$; some insurance applications of Sarmanov distributions are illustrated in Tang et al. (2011) and Yang and Wang (2012).

Motivated by the underlying relationship between $Q$ and the product $\operatorname{df} Q^{*}=\prod_{i=0}^{n} F_{i}$, in this paper we consider a wider class of multivariate df's which are absolutely continuous with respect to a product df - we refer to that as AC-product class. Specifically, the members of this class are all absolutely continuous df's with respect to $Q^{*}$.

It turns out that under some weak conditions the asymptotic behaviour of the aggregated risk $S$ and the deflated risk $Z$ for risks with an AC-product distribution can be derived explicitly.

Organization of the rest of the paper: In the next section we briefly discuss some basic properties of AC-product distributions. Further, we derive a novel result concerning the Sarmanov distribution, which is the canonical example of the AC-product class. Section 3 shows the asymptotic independence of AC-product risks, whereas Section 4 investigates the asymptotic behaviour of the deflated risk $Z$ under extreme value type conditions on the marginal df's. In Section 5 we present three applications concerning risk aggregation, Value-at-Risk and conditional tail expectation, and the probability of ruin under risky investment. The proofs of all the results are postponed to Section 6.

\section{Multivariate AC-Product and Sarmanov Distributions}

In this section we present some details on the class of AC-product distributions and Sarmanov distributions. Hereafter $\boldsymbol{X}=\left(X_{0}, X_{1}, \ldots, X_{n}\right)$ is a $(n+1)$-dimensional random vector with non-negative univariate marginal df's $F_{i}, 0 \leq i \leq n$. It is not standard to write the first component of $\boldsymbol{X}$ by $X_{0}$; we do this since this component will be a reference one in the part when the products of the components of $\boldsymbol{X}$ are discussed. Clearly, if $\boldsymbol{X}$ possesses the df $Q^{*}=\prod_{i=0}^{n} F_{i}$, then the random vector $\boldsymbol{X}$ has independent components, a situation which is often not encountered in practical applications. Starting from this independence setup, a tractable dependence 
structure is introduced by considering $\boldsymbol{X}$ such that its $\mathrm{df} Q$ is absolutely continuous with respect to the product df $Q^{*}$ i.e.,

$$
Q\left(x_{0}, \ldots, x_{n}\right)=\int_{0}^{x_{0}} \cdots \int_{0}^{x_{n}} \eta\left(s_{0}, \ldots, s_{n}\right) Q^{*}\left(\mathrm{~d} s_{0}, \ldots, \mathrm{d} s_{n}\right), \quad x_{i} \in\left[0, \hat{x}_{i}\right], 0 \leq i \leq n,
$$

where $\eta(\cdot)$ is a non-negative measurable function, i.e., if we write $(2.1)$ as

$$
\mathrm{d} Q=\eta \cdot \mathrm{d} Q^{*},
$$

we see that $\eta$ is the Radon-Nikodym derivative. Throughout this paper

$$
X_{0}^{*}, \ldots, X_{n}^{*}
$$

are independent rv's with df's $F_{i}, 0 \leq i \leq n$, respectively, and thus joint df $Q^{*}$. We refer to $Q$ as an AC-product distribution. Since $Q$ is a proper df we shall assume that

$$
\mathbb{E}\left\{\eta\left(X_{x_{0}}^{*}, \ldots, X_{x_{n}}^{*}\right)\right\}<\infty
$$

almost surely with respect to $Q^{*}$ where $X_{x_{i}}^{*}=X_{i}^{*}$ or $X_{x_{i}}^{*}=x_{i}$ with $x_{i}$ in the support of $F_{i}$. Further, we suppose that

$$
\mathbb{E}\left\{\eta\left(X_{0}^{*}, \ldots, X_{n}^{*}\right)\right\}=1
$$

holds. Clearly, (2.2) is satisfied when $\eta(\cdot)$ is a bounded function.

The Sarmanov distributions mentioned in the Introduction are obtained when

$$
\eta\left(x_{0}, \ldots, x_{n}\right)=1+\sum_{0 \leq k<l \leq n} \theta_{k l} \phi_{k}\left(x_{k}\right) \phi_{l}\left(x_{l}\right),
$$

with $\phi_{0}, \ldots, \phi_{n}$ some given real-valued kernels, and $\theta_{k l}, 0 \leq k<l \leq n$ non-negative constants.

In order for such $\eta(\cdot)$ to define a proper df, we shall impose the following assumptions on the kernels:

A1. $\phi_{i}, 0 \leq i \leq n$ are not identical to 0 in $\left[0, \hat{x}_{i}\right]$;

A2. for all $x_{i} \in\left[0, \hat{x}_{i}\right], 0 \leq i \leq n$ we have

$$
\sum_{0 \leq k<l \leq n} \theta_{k l} \phi_{k}\left(x_{k}\right) \phi_{l}\left(x_{l}\right) \geq-1
$$

almost surely with respect to $Q^{*}$;

A3. for any $0 \leq i \leq n$ we have

$$
\mathbb{E}\left\{\phi_{i}\left(X_{i}\right)\right\}=0 .
$$

Apart form the choice $\phi_{i}=1-2 F_{i}$ which leads to the FGM distribution, another common specification of the kernels is $\phi_{i}(s)=g_{i}(s)-\mathbb{E}\left\{g_{i}\left(X_{i}\right)\right\}, s>0$, for some function $g_{i}$ such that $\mathbb{E}\left\{g_{i}\left(X_{i}\right)\right\}<\infty$.

We may consider for instance $g_{i}(s)=\exp (-s)$, or $g_{i}(s)=s^{\alpha_{i}}, \alpha_{i} \in \mathbb{R}$, provided that $\mathbb{E}\left\{X_{i}^{\alpha_{i}}\right\}<\infty$ and $\hat{x}_{i}<\infty$. The next lemma shows that the kernels need to obey certain asymptotic restrictions.

Lemma 2.1. Let $Q$ be a $(n+1)$-dimensional multivariate Sarmanov distribution of $\left(X_{0}, \ldots, X_{n}\right)$ with $\eta$ defined by the kernel functions $\phi_{i}, 0 \leq i \leq n$ and non-negative weights $\theta_{k l}, 0 \leq k<l \leq n$, as in (2.4). Suppose that $\phi_{i}$ is continuous at both $0, \hat{x}_{i}$ and bounded on finite intervals of $\left(0, \hat{x}_{i}\right)$. If further A1-A3 hold, then

$$
\sup _{x \in\left[0, \hat{x}_{i}\right]}\left|\phi_{i}(x)\right|<M_{i}<\infty \quad 0 \leq i \leq n
$$

holds for some positive constants $M_{0}, \ldots, M_{n}$. 
In the light of Lemma 2.1 if the df $\widetilde{F}_{i}$ is such that

$$
1-\widetilde{F}_{i}(x)=\int_{x}^{\hat{x}_{i}}\left(1-\frac{\phi_{i}(u)}{M_{i}}\right) F_{i}(\mathrm{~d} u), \quad 0 \leq x<\hat{x}_{i}, 0 \leq i \leq n,
$$

then $\widetilde{F}_{i}$ are proper univariate df's. For each $0 \leq i \leq n$, by $(2.6), \widetilde{F}_{i}$ is a proper df with the same upper endpoint $\hat{x}_{i}$ as the df $F_{i}$.

Lemma 2.1 motivates the following assumptions on the kernel functions

$$
\lim _{x \uparrow \hat{x}_{i}} \phi_{i}(x)=\kappa_{i} \in \mathbb{R}, \quad 0 \leq i \leq n
$$

which implies that for any $M_{i}>\kappa_{i}$

$$
1-\widetilde{F}_{i}(x) \sim\left(1-\frac{\kappa_{i}}{M_{i}}\right) \overline{F_{i}}(x), \quad x \uparrow \hat{x}_{i}
$$

In this paper $\sim$ means asymptotic equivalence, i.e., the quotient of both sides tend to 1 . A consequence of condition (2.9) is that

$$
\lim _{x_{i} \uparrow \hat{x}_{i}, i=0, \ldots, n}\left(1+\sum_{0 \leq k<l \leq n} \theta_{k l} \phi_{k}\left(x_{k}\right) \phi_{l}\left(x_{l}\right)\right)=1+\sum_{0 \leq k<l \leq n} \theta_{k l} \kappa_{k} \kappa_{l}=: \Lambda_{-} .
$$

Since $\eta(\cdot)$ is non-negative, then also $\Lambda_{-}$is non-negative; if we do not explicitly specify the Radon-Nikodyn derivative of $Q$ the natural extension of the above is to require that

$$
\lim _{x_{i} \uparrow \hat{x}_{i}, i=0, \ldots, n} \eta\left(x_{0}, \ldots, x_{n}\right)=\Lambda_{-} \in[0, \infty) .
$$

\section{Asymptotic Independence}

In various insurance applications, see e.g., Asimit et al. (2011) it is crucial to find concrete multivariate distributions which possess certain asymptotic dependence properties. As mentioned above, the FGM distribution is included in the class of Sarmanov distributions; Hashorva and Hüsler (1999) shows that these distributions have asymptotically independent marginals (see below for the definition), and therefore the maxima of multivariate random samples with underlying FGM distribution have asymptotically independent components, provided that each marginal distribution is in the max-domain of attraction (MDA) of some univariate df. In order to give more precise statements, we briefly mention that a univariate df $F$ on $\mathbb{R}$ belongs to the MDA of a univariate extreme value df $N$, denoted by $F \in \operatorname{MDA}(N)$, if

$$
\lim _{n \rightarrow \infty} \sup _{x \in \mathbb{R}}\left|F^{n}\left(c_{n} x+d_{n}\right)-N(x)\right|=0
$$

holds for constants $c_{n}>0$ and $d_{n} \in \mathbb{R}, n \geq 1$.

Only three candidates for the df $N$ are possible, namely the Fréchet distribution $\Phi_{\gamma}$, the Gumbel distribution $\Lambda$ and the Weibull distribution $\Psi_{\gamma}$, where $\gamma>0$ indexes members of the Fréchet and Weibull families. We mention some basic properties of univariate extreme value distributions and their MDA below; see Resnick (1987) or Embrechts et al. (1997) for more details.

When $Q$ is the $\mathrm{df}$ of the bivariate random vector $\left(X_{1}, X_{2}\right)$ with marginal df's $F_{1}$ and $F_{2}$ such that $F_{i} \in$ $\operatorname{MDA}\left(N_{i}\right), i=1,2$, then we say that $Q$ has asymptotically independent marginal distributions if for any positive $x, y$

$$
\lim _{n \rightarrow \infty} n \mathbb{P}\left(X_{1}>c_{n 1} x+d_{n 1}, X_{2}>c_{n 2} y+d_{n 2}\right)=0,
$$

where $c_{n i}>0, d_{n i}, n \geq 1, i=1,2$ are constants such that $F_{i} \in \operatorname{MDA}\left(N_{i}\right)$ holds as given by (3.1). In order to simplify the presentation we abbreviate the above as $Q \in \operatorname{MDA}\left(N_{1} ; N_{2}\right)$.

Next, we consider $Q$ a bivariate distribution functions such that it has marginal distributions $F_{1}, F_{2}$ and a positive Radon-Nikodyn density $\eta(\cdot)$ with respect to $F_{1} \cdot F_{2}$. The general case of a $d$-dimensional distribution 
follows easily since pair-wise asymptotic independence implies asymptotic independence of the multivariate distributions.

Proposition 3.1. Let $Q$ be a bivariate $d f$ as above with marginal $d f$ 's $F_{1}$ and $F_{2}$ such that $F_{i} \in \operatorname{MDA}\left(N_{i}\right), i=$ 1,2. If further

$$
\limsup _{s \uparrow \hat{x}_{1}, t \uparrow \hat{x}_{2}} \eta(s, t)<\infty
$$

then $Q \in \operatorname{MDA}\left(N_{1} ; N_{2}\right)$.

By combining the above result with Lemma 2.1 we obtain:

Corollary 3.2. If $Q$ is a multivariate Sarmanov distribution such that the kernels satisfy the assumptions of Lemma 2.1, then $Q$ has asymptotically independent marginal distributions and it belongs to the max-domain of attraction of a product max-stable distribution provided that $F_{i} \in M D A\left(N_{i}\right), 0 \leq i \leq n$ with $N_{i}$ some univariate extreme value distribution.

\section{Extreme Value Risk Models}

In various insurance and finance applications the investigation of the tail asymptotics of products is a crucial task, see e.g., Berman (1992), Cline and Samorodnitsky (1994), Jessen and Mikosch (2006), Tang (2006a,b,2008), Hashorva and Pakes (2010), Hashorva et al. (2010,2011,2012), Liu and Tang (2010), Arendarczyk and Dębicki (2011,2012), Constantinescu et al. (2011), Yang et al. (2011), Hashorva (2011,2012), Yang and Wang (2012). Specifically, if $X_{0}, \ldots, X_{n}$ are non-negative rv's modeling some risks, then it is of interest to investigate the tail asymptotics of the deflated risk $Z=\prod_{i=0}^{n} X_{i}$ with df $H$. When $X_{i}, 0 \leq i \leq n$ are mutually independent, using extreme value theory, it is possible to obtain some explicit results. A classical case is when $X_{0}$ has a regularly varying survival function and the other rv's satisfy certain moment conditions which allow to use Breiman's lemma (see Breiman (1965) and the recent results of Yang and Wang (2012)). Recall that a univariate df $F$ has a regularly varying survival function $\bar{F}$ with index $-\gamma \leq 0$, if for any $y \in(0, \infty)$

$$
\lim _{x \rightarrow \infty} \frac{\bar{F}(x y)}{\bar{F}(x)}=y^{-\gamma}
$$

It is well-known that when $\gamma>0$, then (4.1) is equivalent with $F \in \operatorname{MDA}\left(\Phi_{\gamma}\right)$, see e.g., Mikosch (2009). Note that $\Phi_{\gamma}(x)=\exp \left(-x^{-\gamma}\right), x>0$, and necessarily, for the Fréchet case we have that the upper endpoint of $F$ is infinite. For a univariate df $F$ with a finite upper endpoint $\hat{x} \in(0, \infty)$ we are interested on its asymptotic behaviour at $\hat{x}$. Instead of (4.1) we shall assume for this case that $\bar{F}$ is regularly varying at $\hat{x}$ with index $\gamma \geq 0$ i.e.,

$$
\lim _{x \rightarrow \infty} \frac{\bar{F}(\hat{x}-y / x)}{\bar{F}(\hat{x}-1 / x)}=y^{\gamma}
$$

for any $y>0$. When $\gamma>0$ the above condition is equivalent with $F$ is in the MDA of the Weibull df $\Psi_{\gamma}$ (we recall $\left.\Psi_{\gamma}(x)=\exp \left(-|x|^{\gamma}\right), x<0\right)$.

If otherwise specified, in the sequel we assume that $\boldsymbol{X}=\left(X_{0}, X_{1}, \ldots, X_{n}\right)$ has the $\mathrm{df} d Q=\eta \cdot d Q^{*}$, where $Q^{*}=\prod_{i=0}^{n} F_{i}$ is a product df with non-degenerate univariate df's $F_{0}, \ldots, F_{n}$. We state next the first result of this section, the case of Gumbel MDA is treated in Theorem 4.2 below. Recall that $\left(X_{0}^{*}, \ldots, X_{n}^{*}\right)$ has $\mathrm{df} Q^{*}$. 
Theorem 4.1. a) Suppose that for each fixed $x_{1}, \ldots, x_{n} \geq 0$ and $\bar{\eta}(\cdot)$ some bounded function

$$
\lim _{x_{0} \rightarrow \infty} \eta\left(x_{0}, x_{1}, \ldots, x_{n}\right)=\bar{\eta}\left(x_{1}, \ldots, x_{n}\right)
$$

If further $\overline{F_{0}}$ satisfies (4.1) with some $\gamma \geq 0$ and $\mathbb{E}\left\{X_{i}^{\gamma+\epsilon}\right\}<\infty$ holds for all $1 \leq i \leq n$ and some $\epsilon>0$, then the survival function $\bar{H}$ of $Z$ satisfies (4.1) and moreover as $x \rightarrow \infty$

$$
\bar{H}(x) \sim \overline{F_{0}}(x) \mathbb{E}\left\{\bar{\eta}\left(X_{1}^{*}, \ldots, X_{n}^{*}\right) \prod_{i=1}^{n}\left(X_{i}^{*}\right)^{\gamma}\right\} .
$$

b) Suppose that $F_{i}, 0 \leq i \leq n$ satisfy (4.2) with $\gamma_{i} \geq 0,0 \leq i \leq n$. If (2.12) holds with $\Lambda_{-}>0$, then as $x \uparrow \prod_{i=0}^{n} \hat{x}_{i}$

$$
\bar{H}(x) \sim \frac{\Lambda_{-}}{\Gamma\left(\sum_{i=0}^{n} \gamma_{i}+1\right)} \prod_{i=0}^{n}\left(\Gamma\left(\gamma_{i}+1\right) \overline{F_{i}}\left(\hat{x}_{i} \bar{x}\right)\right),
$$

where $\bar{x}=x \prod_{i=0}^{n} \hat{x}_{i}^{-1}$, and $\Gamma(\cdot)$ is the Euler gamma function.

Applied to the case of Sarmanov distributions, Theorem 4.1 a) implies the following result:

Corollary 4.1. Under the assumptions of statement a) of Theorem 4.1, if further $\boldsymbol{X}=\left(X_{0}, X_{1}, \ldots, X_{n}\right)$ follows a multivariate Sarmanov distribution of the form (2.1) such that the assumptions of Lemma 2.1 hold, then as $x \rightarrow \infty$

$$
\bar{H}(x) \sim \overline{F_{0}}(x) \prod_{i=1}^{n} \mathbb{E}\left\{X_{i}^{\gamma}\right\}\left(1+\kappa_{0} \sum_{1 \leq l \leq n} \theta_{0 l} \frac{\mathbb{E}\left\{X_{l}^{\gamma} \phi_{l}\left(X_{l}\right)\right\}}{\mathbb{E}\left\{X_{l}^{\gamma}\right\}}+\sum_{1 \leq k<l \leq n} \theta_{k l} \frac{\mathbb{E}\left\{X_{k}^{\gamma} \phi_{k}\left(X_{k}\right)\right\} \mathbb{E}\left\{X_{l}^{\gamma} \phi_{l}\left(X_{l}\right)\right\}}{\mathbb{E}\left\{X_{k}^{\gamma}\right\} \mathbb{E}\left\{X_{l}^{\gamma}\right\}}\right)
$$

provided that $\lim _{x \rightarrow \infty} \phi_{0}(x)=\kappa_{0} \in \mathbb{R}$.

Remarks: i) For the case of bivariate FGM distributions Jiang and Tang (2011) obtained the tail asymptotic of $Z$, see also Yang et al. (2011).

ii) Yang and Wang (2012) derived (4.6) under weaker conditions for the bivariate setting of Sarmanov distributions.

In various applications due for instance to different currencies, original risks are linearly transformed. In order to widen the applications to those cases, suppose therefore that $X_{i}, 0 \leq i \leq n$ has df with lower endpoint equal 0 (i.e., $\left.\inf \left\{x \in \mathbb{R}: F_{i}(x)>0\right\}=0\right)$, and let the random vector $\boldsymbol{Y}=\left(Y_{0}, \ldots, Y_{n}\right)$ be such that

$$
Y_{i}=\left(a_{i}+b_{i} X_{i}\right)^{-1}, \quad a_{i}>0, b_{i}>0,0 \leq i \leq n
$$

The df $Q_{\boldsymbol{Y}}$ of $\boldsymbol{Y}$ is related to $Q_{\boldsymbol{Y}}^{*}:=\prod_{i=0}^{n} F_{Y_{i}}$ by $\mathrm{d} Q_{\boldsymbol{Y}}=\eta_{\boldsymbol{Y}} \cdot \mathrm{d} Q_{\boldsymbol{Y}}^{*}$, where

$$
\eta_{\boldsymbol{Y}}\left(t_{0}, \ldots, t_{n}\right)=\eta\left(b_{0}^{-1}\left(t_{0}^{-1}-a_{0}\right), \ldots, b_{n}^{-1}\left(t_{n}^{-1}-a_{n}\right)\right) .
$$

The following result is a consequence of statement b) of Theorem 4.1.

Corollary 4.2. If $F_{i}, 0 \leq i \leq n$ is regularly varying at zero with index $\gamma_{i} \geq 0$, and further

$$
\lim _{x_{i} \downarrow 0, i=0, \ldots, n} \eta\left(x_{0}, \ldots, x_{n}\right)=\eta(0+, \ldots, 0+)=: \Lambda_{+} \in(0, \infty),
$$

then as $x \uparrow \prod_{i=0}^{n} a_{i}^{-1}$

$$
\mathbb{P}\left(\prod_{i=0}^{n} Y_{i}>x\right) \sim \frac{\Lambda_{+}}{\Gamma\left(\sum_{i=0}^{n} \gamma_{i}+1\right)} \prod_{i=0}^{n}\left(\Gamma\left(\gamma_{i}+1\right) F_{i}\left(b_{i}^{-1}\left(y_{i}^{-1}-a_{i}\right)\right)\right), \quad y_{i}:=x \prod_{j=0, j \neq i}^{n} a_{i} .
$$


Next, our main assumption on $F_{0}$ is that it belongs to Gumbel MDA; we recall that when in $(3.1) N(x)=$ $\Lambda(x)=\exp \left(-e^{-x}\right), x \in \mathbb{R}$, then an equivalent condition for (3.1) to hold is

$$
\lim _{x \uparrow \hat{x}} \frac{\bar{F}(x+y a(x))}{\bar{F}(x)}=e^{-y}, \quad \forall y \geq 0,
$$

with some positive scaling function $a(\cdot)$. When (4.10) holds we shall use the abbreviation $F \in \operatorname{MDA}(\Lambda, a(\cdot))$. The scaling function $a(\cdot)$ satisfies

$$
\lim _{x \uparrow \hat{x}} \frac{x}{a(x)}=\infty \text {, and } \lim _{x \uparrow \hat{x}} \frac{\hat{x}-x}{a(x)}=\infty \quad \text { if } \hat{x}<\infty .
$$

Theorem 4.2. If $F_{0} \in \operatorname{MDA}(\Lambda, a(\cdot))$ and further $F_{i}, 1 \leq i \leq n$ satisfy (4.2) with non-negative constants $\gamma_{i}, 1 \leq i \leq n$, then as $x \uparrow \prod_{i=0}^{n} \hat{x}_{i}$

$$
\bar{H}(x) \sim \Lambda_{-} \overline{F_{0}}(\widetilde{x}) \prod_{i=1}^{n}\left(\Gamma\left(\gamma_{i}+1\right) \bar{F}_{i}\left(\hat{x}_{i}-\frac{\hat{x}_{i} a(\widetilde{x})}{\widetilde{x}}\right)\right), \quad \widetilde{x}:=x \prod_{i=1}^{n} \hat{x}_{i}^{-1} .
$$

Remarks: i) The Gumbel MDA assumption on $F_{0}$ and the assumptions on $F_{i}, 1 \leq i \leq n$ imply that $F_{0}\left(\hat{x}_{0}-\right)=$ $\cdots=F_{n}\left(\hat{x}_{n}-\right)=0$. Consider for simplicity $n=1$ and $\mathbb{P}\left(X_{1}=\hat{x}_{1}\right)=p_{1}>0$. Then under the assumptions of Theorem 4.2 for $F_{0}$, we obtain as $x \uparrow \hat{x}_{0} \hat{x}_{1}$

$$
\bar{H}(x) \sim \Lambda_{-} p_{1} \overline{F_{0}}\left(\hat{x}_{1}^{-1} x\right) .
$$

ii) Since uniformly with respect to $z$ in every compact set of $\mathbb{R}$

$$
\lim _{u \uparrow \hat{x}} \frac{a(u+z a(u))}{a(u)}=1
$$

it follows that $H$ is in the Gumbel MDA with the same scaling function $a(\cdot)$ as $F_{0}$.

\section{Applications}

5.1. Asymptotics of CTE and VaR. For this application we regard $X_{0}$ as a base rv which models an insurance risk, and $X_{1}, \ldots, X_{n}$ as random deflators of the base risk. In various applications, say for instance in risk management, the deflated risk $Z=X_{0} \prod_{i=1}^{n} X_{i}$ needs to be investigated. We write as above $F_{i}, 1 \leq i \leq n$ for the $\mathrm{df}$ of $X_{i}$ and assume that $F_{0}$ is continuous. Due to regulatory restrictions, it is of actuarial interest to quantify the asymptotic behaviour of $\operatorname{VaR}_{Z}(p)$ (Value-at-Risk) and $\mathrm{CTE}_{Z}(p)$ (conditional tail expectation) as $p \rightarrow 1$. We recall that

$$
\operatorname{CTE}_{Z}(p):=\mathbb{E}\left\{Z \mid Z>\operatorname{VaR}_{Z}(p)\right\}=\mathbb{E}\left\{Z-\operatorname{VaR}_{Z}(p) \mid Z>\operatorname{VaR}_{Z}(p)\right\}+\operatorname{VaR}_{Z}(p), \quad p \in(0,1),
$$

where

$$
\operatorname{VaR}_{Z}(p):=\inf \{x: \mathbb{P}(Z \leq x) \geq p\}, \quad p \in(0,1) .
$$

See Denuit et al. (2006) for the basic properties of VaR and CTE. When the base risk $X_{0}$ is dependent to $X_{i}, 1 \leq i \leq n$ such that the assumptions of Theorem 4.2 hold, then by (4.14) $Z$ has its df in the Gumbel MDA with the same scaling function as $X_{0}$. Since the scaling function $a(\cdot)$ is asymptotically equivalent to the mean excess function, we may write (see also Asimit and Badescu (2010))

$$
\lim _{p \uparrow 1} \frac{\mathbb{E}\left\{Z-\operatorname{VaR}_{Z}(p) \mid Z>\operatorname{VaR} R_{Z}(p)\right\}}{\mathbb{E}\left\{X_{0}-\operatorname{VaR}_{X_{0}}(p) \mid X_{0}>\operatorname{VaR}_{X_{0}}(p)\right\}}=1,
$$

and consequently,

$$
\lim _{p \uparrow 1} \frac{\operatorname{CTE}_{X_{0}}(p)}{V a R_{X_{0}}(p)}=\lim _{z \uparrow \prod_{i=0}^{n} \hat{x}_{i}} \frac{\operatorname{CTE}_{Z}(z)}{z}=1
$$


It is well-known that for continuous risks CTE is more conservative than Value at Risk (VaR), which is the quantile function of the random variable of interest, if both are evaluated at the same confidence level $p$ (see also the CTE definition from above). In the next result we show that Value-at-Risk for $p$ close to 1 is asymptotically the same for $Z$ and $X_{0}$.

Theorem 5.1. Under the assumptions of Theorem 4.2 we have

$$
\mathrm{CTE}_{Z}(p) \sim \operatorname{VaR}_{Z}(p) \sim \operatorname{VaR}_{X_{0}}(p) \sim \operatorname{CTE}_{X_{0}}(p)
$$

as $p \uparrow 1$.

The above theorem shows that both risk measures VaR and CTE for the base risk $X_{0}$ and the product risk $Z$ are asymptotically equivalent, provided that the underlying dependence structure of the risks is determined by an AC-product df; see Hashorva et al. (2010) for the case of independent risks.

5.2. Risk Aggregation. A key topic in insurance and finance with diverse applications in risk management is the risk aggregation, see e.g., Goovaerts et al. (2005), Geluk and Tang (2009), Asimit et al. (2011), Hashorva (2013a), Kortschak and Hashorva (2013) and the references therein. Asymptotic considerations for the investigation of the total (or aggregated) risk turn out to be quite important when risks are dependent. As already shown in Section 2 the dependence structure of the risks therein exhibit asymptotic independence under a mild condition on the density function $\eta(\cdot)$. For such risks, the Mitra-Resnick methodology developed in Mitra and Resnick (2009) is powerful for deriving exact asymptotic results for the tail of aggregated risk. If $X_{0}, \ldots, X_{n}$ are independent, then under various conditions the aggregated risk $S=\sum_{i=0}^{n} X_{i}$ has a tractable tail asymptotic behaviour. When $X_{0}$ has the df in the Gumbel MDA, under the Mitra-Resnick framework the tail asymptotic behaviour of $S$ is determined by the tail asymptotics of one component, say $X_{0}$. In our application below we are able to describe the effect of a single component on the aggregated risk for the risk structures dealt with here. Specifically, let $\left(X_{0}, \ldots, X_{n}\right)$ has the joint df $Q$ given by

$$
\mathrm{d} Q=\eta \cdot \mathrm{d} Q^{*}, \quad Q^{*}=\prod_{i=0}^{n} F_{i},
$$

with $F_{i}$ marginal distributions with support on $[0, \infty)$. If $Q_{i j}$ is the df of $\left(X_{i}, X_{j}\right)$, then by (2.2) we have $\mathrm{d} Q_{i j}=\eta_{i j} \cdot \mathrm{d}\left(F_{i} F_{j}\right), i \neq j$. In view of Proposition 3.1 when

$$
\limsup _{s \uparrow \hat{x}_{i}, t \uparrow \hat{x}_{j}} \eta_{i j}(s, t)<\infty,
$$

then $Q_{i j}$ has asymptotically independent marginals, provided that $F_{i}$ and $F_{j}$ are in some MDA of a univariate extreme value df. In the next theorem we shall assume that only $F_{0}$ is in the Gumbel MDA with some scaling function $a(\cdot)$ and upper endpoint $\hat{x}_{0}=\infty$; all the df's $F_{i}, 0 \leq i \leq n$ satisfy

$$
\lim _{x \rightarrow \infty} \frac{\overline{F_{i}}(x)}{\mathbb{P}\left(X_{0}>x\right)}=c_{i} \in[0, \infty), \quad 0 \leq i \leq n .
$$

Theorem 5.2. Let $\left(X_{0}, \ldots, X_{n}\right)$ be as above, and assume that $F_{0} \in \operatorname{MDA}(\Lambda, a(\cdot))$ and both (5.4), (5.5) are satisfied. If further $\lim _{x \rightarrow \infty} a(x)=\infty$ and for positive constants $L_{i j}, 1 \leq i<j \leq n$

$$
\lim _{x \rightarrow \infty} \max _{0 \leq i<j \leq n} \frac{\overline{F_{i}}\left(a(x) L_{i j}\right) \overline{F_{j}}\left(a(x) L_{i j}\right)}{\overline{F_{0}}(x)}=0,
$$

then as $x \rightarrow \infty$

$$
\mathbb{P}(S>x) \sim \mathbb{P}\left(X_{0}>x\right) \sum_{i=0}^{n} c_{i}
$$


and $S$ has $d f$ in the Gumbel MDA with the same scaling function $a(\cdot)$ as $F_{0}$.

Clearly, when all marginal distributions are tail equivalent to $F_{0}$, then condition (5.6) is the Mitra-Resnick condition for the aggregation of independent risks, see (2.15) in Mitra and Resnick (2009).

5.3. Ruin in the Presence of Risky Investments. Our next application concerns the discrete-time insurance risk model discussed in Hashorva et al. (2010) and Tang et al. (2011). Let in the following $R_{1}, \ldots, R_{n}$ be independent real-valued rv's with common df $F$ being further independent of $\Delta_{1}, \ldots, \Delta_{n}$, whose support is $(-1, \infty)$. Several authors have considered the asymptotic behaviour as $u \rightarrow \infty$ of the following ruin probability

$$
\psi(u ; n)=\mathbb{P}\left(\min _{0 \leq i \leq n} U_{i}<0 \mid U_{0}=u\right), \quad U_{0}=u \geq 0,
$$

where

$$
\Upsilon_{i}\left(U_{i}+R_{i}\right)=U_{i-1}, \quad 1 \leq i \leq n,
$$

with $\delta_{i}>0, p_{i} \in[0,1), 1 \leq i \leq n$ and

$$
\Upsilon_{i}:=\left(c_{i}+p_{i}\left(1+\Delta_{i}\right)\right)^{-1}, \quad c_{i}:=\left(1-p_{i}\right)\left(1+\delta_{i}\right) .
$$

In the light of Theorem 5.1 in Tang et al. (2011) we have when the df $F$ belongs to the well-known subexponential class

$$
\psi(u ; n) \sim \sum_{k=1}^{n} \mathbb{P}\left(R \prod_{i=1}^{k} \Upsilon_{i}>u\right), \quad u \rightarrow \infty,
$$

where $\Upsilon_{1}, \ldots, \Upsilon_{n}$ can be arbitrarily dependent. We note in passing that a df $F$ on $[0, \infty)$ is said to be subexponential, written as $F \in \mathcal{S}$, if $\overline{F^{2 *}}(x) \sim 2 \bar{F}(x)$ as $x \rightarrow \infty$, where $F^{2 *}$ denotes the two-fold convolution of $F$; more generally, $F$ on $\mathbb{R}$ is still said to be subexponential if the $\mathrm{df} F_{+}(x)=F(x) \mathbf{1}_{\{x \geq 0\}}$ is subexponential, see e.g., Embrechts et al. (1997).

Hashorva et al. (2010) discussed the case $\Upsilon_{i}, 1 \leq i \leq n$ are independent, whereas the recent paper Tang et al. (2011) obtained some refinements of (5.8), which allow for dependence assuming a multivariate Sarmanov distribution for $\left(1+\Delta_{1}, \ldots, 1+\Delta_{n}\right)$, see Theorem 4.1 and 4.2 in Tang et al. (2011).

Two recent papers Chen (2011) and Yang and Wang (2012) investigated a similar case where $\left(R_{i}, \Upsilon_{i}\right), 1 \leq i \leq n$ are independent and for each $1 \leq i \leq n,\left(R_{i}, \Upsilon_{i}\right)$ follows a bivariate FGM or Sarmanov distribution, respectively, see Theorem 3.1 and Corollary 3.1 in Chen (2011) and Theorem 4.1 in Yang and Wang (2012). As we show below, it is possible to obtain similar results as Theorem 4.1 and 4.2 in Tang et al. (2011) by considering a more general dependence structure.

Next, we assume that $R_{1}, \ldots, R_{n}$ are independent real-valued rv's, and $\left(\Upsilon_{1}, \ldots, \Upsilon_{n}\right)$, independent of $R_{1}, \ldots, R_{n}$, has an AC-product distribution. Assume therefore that $\mathbf{\Upsilon}=\left(\Upsilon_{1}, \ldots, \Upsilon_{n}\right)$ has the joint $\mathrm{df}$

$$
\mathrm{d} G_{\Upsilon}=\eta_{\Upsilon} \cdot \mathrm{d} G^{*}, \quad G^{*}=\prod_{i=1}^{n} G_{i},
$$

where $G_{i}$ is the df of $\Upsilon_{i}, 1 \leq i \leq n$. Thus, for each $1 \leq k<n, \Upsilon_{\mathbf{k}}=\left(\Upsilon_{1}, \ldots, \Upsilon_{k}\right)$ has the joint df given by $\eta_{\Upsilon_{\mathbf{k}}} \cdot \prod_{i=1}^{k} G_{i}$ with

$$
\eta_{\Upsilon_{\mathbf{k}}}\left(x_{1}, \ldots, x_{k}\right)=\int_{0}^{c_{k+1}^{-1}} \ldots \int_{0}^{c_{n}^{-1}} \eta_{\Upsilon}\left(x_{1}, \ldots, x_{n}\right) \prod_{i=k+1}^{n} G_{i}\left(\mathrm{~d} x_{i}\right) .
$$


Theorem 5.3. Consider the discrete-time risk model introduced above with $p_{i} \in[0,1), 1 \leq i \leq n$. Assume that $F \in \operatorname{MDA}(\Lambda, a(\cdot)) \cap \mathcal{S}$ and $G_{i}, 1 \leq i \leq n$ satisfy (4.2) at $c_{i}^{-1}$ with non-negative constants $\gamma_{i}, 1 \leq i \leq n$. If for each $1 \leq k \leq n$ the limit $\Lambda_{\Upsilon_{\mathbf{k}}-}=\lim _{x_{i} \uparrow c_{i}^{-1}, i=1, \ldots, k} \eta_{\Upsilon_{\mathbf{k}}}\left(x_{1}, \ldots, x_{k}\right)$ exists and is finite, then as $u \rightarrow \infty$

$$
\psi(u ; n) \sim \bar{F}(\widetilde{u}) \sum_{k=1}^{n} \Lambda_{\Upsilon_{\mathrm{k}}}-\prod_{i=1}^{k}\left(\Gamma\left(\gamma_{i}+1\right) \overline{G_{i}}\left(c_{i}^{-1}-\frac{c_{i}^{-1} a(\widetilde{u})}{\widetilde{u}}\right)\right)
$$

where $\widetilde{u}=u \prod_{i=1}^{n} c_{i}$.

Note in passing that if $F \in \operatorname{MDA}(\Lambda, a(\cdot))$ with $a(\cdot)$ such that $\lim _{u \rightarrow \infty} a(u)=\infty$, then in order to show that $F \in \mathcal{S}$ we can utilise the criteria given in Hashorva et al. (2010).

In the literature, there are several results concerned with the tail asymptotic behaviour of randomly weighted sums with unbounded weights, that is, $p_{i}, 1 \leq i \leq n$ can be 1 , see e.g., Resnick and Willekens (1991). Next, we consider only the case that $\bar{F}$ is regularly varying at infinity.

Theorem 5.4. Consider the discrete-time risk model introduced above with $p_{i} \in[0,1], 1 \leq i \leq n$. Assume that $F$ satisfies (4.1) with some $\gamma \geq 0$ and for each $1 \leq i \leq n$ and $\epsilon>0$ we have $\mathbb{E}\left\{\Upsilon_{i}^{\gamma+\epsilon}\right\}<\infty$. If the function $\eta_{\Upsilon}$ is bounded, then as $u \rightarrow \infty$

$$
\psi(u ; n) \sim \mathcal{K} \bar{F}(u)
$$

where $\mathcal{K}:=\sum_{k=1}^{n} \mathbb{E}\left\{\eta_{\Upsilon_{\mathbf{k}}}\left(\Upsilon_{1}^{*}, \ldots, \Upsilon_{k}^{*}\right) \prod_{i=1}^{k}\left(\Upsilon_{i}^{*}\right)^{\gamma}\right\} \in(0, \infty)$ and $\Upsilon_{i}^{*}, 1 \leq i \leq n$ are independent such that $\Upsilon_{i}^{*}$ and $\Upsilon_{i}$ have the same df for each $1 \leq i \leq n$.

\section{ProOfs}

Throughout this section, for two positive functions $u(x)$ and $v(x)$, as $x$ tends to $z$, we write $u(x) \sim v(x)$ if $\lim _{x \rightarrow z} u(x) / v(x)=1$; write $u(x)=o(v(x))$ if $\lim _{x \rightarrow z} u(x) / v(x)=0$. Further, write $u(x)=O(v(x))$ if $\limsup _{x \rightarrow z} u(x) / v(x)<\infty$.

Proof of Lemma 2.1.: We show next that $\lim \sup _{x \uparrow \hat{x}_{1}} \phi_{1}(x)=\infty$ is not possible. Let us suppose for a while that this is possible, say for any given $M$ large, in some left-neighbourhood of $\hat{x}_{1}$ we have $\phi_{1}(x)>M$. For any $x \in\left[0, \hat{x}_{1}\right)$ and $y \in\left[0, \hat{x}_{2}\right)$ we obtain

$$
\mathbb{P}\left(X_{1}>x, X_{2}>y\right)=\overline{F_{1}}(x) \overline{F_{2}}(y)+\int_{x}^{\hat{x}_{1}} \phi_{1}(s) F_{1}(\mathrm{~d} s) \int_{y}^{\hat{x}_{2}} \phi_{2}(s) F_{2}(\mathrm{~d} s) .
$$

Since $1+\phi_{1} \phi_{2}$ is the Radon-Nikodyn density of $\left(X_{1}, X_{2}\right)$ with respect to $F_{1} \cdot F_{2}$ we have

$$
1+\phi_{1}(x) \phi_{2}(y) \geq 0, \quad x \in\left[0, \hat{x}_{1}\right), y \in\left[0, \hat{x}_{2}\right)
$$

The above relation and the fact that $M$ can be arbitrarily large imply that $\phi_{2}$ is non-negative (almost surely with respect to the measure generated by $\left.F_{1} F_{2}\right)$. Hence

$$
\overline{F_{1}}(x) \overline{F_{2}}(y)+\int_{x}^{\hat{x}_{1}} \phi_{1}(s) F_{1}(\mathrm{~d} s) \int_{y}^{\hat{x}_{2}} \phi_{2}(s) F_{2}(\mathrm{~d} s)>\overline{F_{1}}(x)
$$

holds for some $x$ close to $\hat{x}_{1}$ and $y$ such that $\int_{y}^{\hat{x}_{2}} \phi_{2}(s) F_{2}(\mathrm{~d} s)>0$. This is a contradiction, since necessarily

$$
\mathbb{P}\left(X_{1}>x, X_{2}>y\right) \leq \min \left(\mathbb{P}\left(X_{1}>x\right), \mathbb{P}\left(X_{2}>y\right)\right)=\min \left(\overline{F_{1}}(x), \overline{F_{2}}(y)\right), \quad x \in\left[0, \hat{x}_{1}\right), y \in\left[0, \hat{x}_{2}\right)
$$

If we assume that $\lim \inf _{x \uparrow \hat{x}_{1}} \phi_{1}(x)=-\infty$, then $\phi_{2}$ cannot be positive and the same argument as above can be repeated to show that this is not possible. 
Next suppose that $\lim \sup _{x \downarrow 0} \phi_{1}(x)=\infty$ is possible. We have

$$
\mathbb{P}\left(X_{1} \leq x, X_{2} \leq y\right)=F_{1}(x) F_{1}(y)+\int_{0}^{x} \phi_{1}(s) F_{1}(\mathrm{~d} s) \int_{0}^{y} \phi_{2}(s) F_{2}(\mathrm{~d} s), \quad x \in\left[0, \hat{x}_{1}\right), y \in\left[0, \hat{x}_{2}\right) .
$$

Hence since again it follows that $\phi_{2}(y)>0$ for some $y \in\left[0, \hat{x}_{2}\right)$, we obtain $F(x, y)>F_{1}(x)$ which is a contradiction. Hence the proof follows showing with similar arguments that $\lim \inf _{x \downarrow 0} \phi_{1}(x)=-\infty$ is also not possible.

Proof of Proposition 3.1.: By the assumptions we have $\lim _{n \rightarrow \infty} c_{n i} x+d_{n i}=\hat{x}_{i}, i=1,2$ for any $x<\hat{x}_{i}$, hence for some $M$ positive and all $n$ large we have (set $\left.u_{n i}(x)=c_{n i} x+d_{n i}, i=1,2\right)$

$$
\begin{aligned}
n \mathbb{P}\left(X_{1}>u_{n 1}(x), X_{2}>u_{n 2}(y)\right) & =n \overline{F_{1}}\left(u_{n 1}(x)\right) \overline{F_{2}}\left(u_{n 2}(y)\right)+n \int_{u_{n 1}(x)}^{\hat{x}_{1}} \int_{u_{n 2}(y)}^{\hat{x}_{2}} \eta(s, t) F_{1}(\mathrm{~d} s) F_{2}(\mathrm{~d} t) \\
& \leq O\left(\overline{F_{1}}\left(u_{n 2}(y)\right)\right)+n M \int_{u_{n 1}(x)}^{\hat{x}_{1}} \int_{u_{n 2}(y)}^{\hat{x}_{2}} F_{1}(\mathrm{~d} s) F_{2}(\mathrm{~d} t) \\
& \rightarrow 0, \quad n \rightarrow \infty,
\end{aligned}
$$

since $F_{1} \cdot F_{2} \in \mathrm{MDA}_{2}\left(N_{1} ; N_{2}\right)$, thus the proof follows.

Proof of Theorem 4.1.: a) Since $F_{0}$ satisfies (4.1), Potter's theorem (see, e.g., Theorem 1.5.6 of Bingham et al. (1987)) implies that for any given $\epsilon>0$ there exist two positive constants $C$ and $D$ such that for all $y>0$ and $x \geq D$

$$
\frac{\overline{F_{0}}(x / y)}{\overline{F_{0}}(x)} \leq C y^{\gamma \pm \epsilon}
$$

For such a constant $D$, we split the survival function $\bar{H}$ of the deflated risk $Z$ into two parts, namely

$$
\begin{aligned}
\bar{H}(x) & =\int_{\left\{x_{i}>0, i=1 \ldots n\right\}}\left(\int_{x \prod_{i=1}^{n} x_{i}^{-1}}^{\infty} \eta\left(x_{0}, x_{1}, \ldots, x_{n}\right) F_{0}\left(\mathrm{~d} x_{0}\right)\right) F_{1}\left(\mathrm{~d} x_{1}\right) \cdots F_{n}\left(\mathrm{~d} x_{n}\right) \\
& =\left(\int_{\left\{\prod_{i=1}^{n} x_{i} \leq x / D\right\}}+\int_{\left\{\prod_{i=1}^{n} x_{i}>x / D\right\}}\right)\left(\int_{x \prod_{i=1}^{n} x_{i}^{-1}}^{\infty} \eta\left(x_{0}, x_{1}, \ldots, x_{n}\right) F_{0}\left(\mathrm{~d} x_{0}\right)\right) F_{1}\left(\mathrm{~d} x_{1}\right) \cdots F_{n}\left(\mathrm{~d} x_{n}\right) \\
& =: J_{1}(x)+J_{2}(x) .
\end{aligned}
$$

Since the function $\eta(\cdot)$ is bounded, then by Markov's inequality and $\mathbb{E}\left\{X_{i}^{\gamma+\epsilon}\right\}<\infty, 1 \leq i \leq n$, we have $x \rightarrow \infty$

$$
J_{2}=O(1) \mathbb{P}\left(\prod_{i=1}^{n} X_{i}^{*}>\frac{x}{D}\right)=O\left(x^{-(\gamma+\epsilon)}\right)=o\left(\overline{F_{0}}(x)\right),
$$

where the last equality holds by (6.1). According to the dominated convergence theorem and by (6.1), under the conditions of the theorem we obtain that

$$
\begin{aligned}
& \lim _{x \rightarrow \infty} \frac{J_{1}(x)}{\overline{\overline{F_{0}}}(x)} \\
& =\int_{\left\{x_{i}>0, i=1 \ldots n\right\}}\left(\lim _{x \rightarrow \infty} \frac{1}{\overline{F_{0}}(x)} \int_{x \prod_{i=1}^{n} x_{i}^{-1}}^{\infty} \eta\left(x_{0}, x_{1}, \ldots, x_{n}\right) F_{0}\left(\mathrm{~d} x_{0}\right) \mathbf{1}_{\left\{\prod_{i=1}^{n} x_{i} \leq x / D\right\}}\right) F_{1}\left(\mathrm{~d} x_{1}\right) \cdots F_{n}\left(\mathrm{~d} x_{n}\right) \\
& =\int_{\left\{x_{i}>0, i=1 \ldots n\right\}} \bar{\eta}\left(x_{1}, \ldots, x_{n}\right)\left(x_{1} \cdots x_{n}\right)^{\gamma} F_{1}\left(\mathrm{~d} x_{1}\right) \cdots F_{n}\left(\mathrm{~d} x_{n}\right) \\
& =\mathbb{E}\left\{\bar{\eta}\left(X_{1}^{*}, \ldots, X_{n}^{*}\right) \prod_{i=1}^{n}\left(X_{i}^{*}\right)^{\gamma}\right\}
\end{aligned}
$$


Consequently, the claim follows from (6.2)-(6.4).

b) By the assumptions, since $\hat{x}_{i}, 0 \leq i \leq n$ is finite, it follows easily that

$$
\bar{H}(x)=\mathbb{P}\left(\prod_{i=0}^{n} X_{i}>x\right) \sim \Lambda_{-} \mathbb{P}\left(\prod_{i=0}^{n} X_{i}^{*}>x\right)
$$

as $x \uparrow \prod_{i=0}^{n} \hat{x}_{i}$, hence the proof is established by a direct application of Lemma 2.1 and Theorem 3.1 in Hashorva et al. (2010).

Proof of Corollary 4.1.: The proof follows by checking the assumptions of Theorem 4.1 which can then be applied to establish the claim.

Proof of Theorem 4.2: We show next the claim for the case $\hat{x}_{0}=\infty$ and omit the proof when $\hat{x}_{0}<\infty$ since it follows with similar arguments. For notational simplicity we assume that $\hat{x}_{i}=1,1 \leq i \leq n$. In view of the Davis-Resnick property of $\bar{H}_{0}$ (see Proposition 1.1 of Davis and Resnick (1988), or details in Hashorva (2013b)) we have that

$$
\lim _{x \rightarrow \infty}\left(\frac{x}{a(x)}\right)^{\mu} \frac{\overline{F_{0}}(\tau x)}{\overline{F_{0}}(x)}=0
$$

holds for any $\mu \geq 0$ and $\tau>1$. Hence for any $c>1$ we have

$$
\bar{H}(x) \sim \mathbb{P}\left(\prod_{i=0}^{n} X_{i}>x, x \leq X_{0} \leq c x, X_{i}>1-1 / c, i=1, \ldots, n\right), \quad x \rightarrow \infty .
$$

Consequently, by the arbitrariness of $c$ we obtain

$$
\bar{H}(x) \sim \Lambda_{-} \mathbb{P}\left(\prod_{i=0}^{n} X_{i}^{*}>x\right), \quad x \rightarrow \infty
$$

and hence the proof follows applying again Theorem 3.1 in Hashorva et al. (2010).

Proof of Theorem 5.1.: In view of (5.2) we need to show that $\operatorname{VaR}_{Z}(p) \sim \operatorname{VaR}_{X_{0}}(p)$ as $p \uparrow 1$. Assume for simplicity that $n=1$ and $\hat{x}_{1}=1$, so we have that $\operatorname{VaR}_{Z}(p) \leq \operatorname{VaR}_{X_{0}}(p)$ as $p \uparrow 1$. If $G=F_{0} F_{1}$ and $G^{-1}, F_{0}^{-1}$ are the generalised inverses of $G$ and $F_{0}$, respectively, then this can be rewritten as $G^{-1}(1-1 / t) \leq F_{0}^{-1}(1-1 / t), t>$ 1. In view of (6.5) both $F_{0}$ and $G$ are in the Gumbel MDA with the same auxiliary function $a(\cdot)$. Consequently (see e.g., Resnick (1987)) $G^{-1}(1-1 / t)$ and $F_{0}^{-1}(1-1 / t)$ are slowly varying functions at infinity. Since further in view of $(6.5)$ for all $x$ large and any $\varepsilon \in(0,1)$ we have

$$
\bar{G}(x) \geq \bar{F}(x(1-\varepsilon)),
$$

then

$$
\lim _{t \rightarrow \infty} \frac{G^{-1}(1-1 / t)}{F_{0}^{-1}(1-1 / t)}=1
$$

and hence the claim follows.

Proof of Theorem 5.2.: Since $\lim _{x \rightarrow \infty} a(x)=\infty$, for all large $x$ and some $M>0$ assumption (5.4) implies for any two different indices $i, j$ and $z>0$

$$
\begin{aligned}
\mathbb{P}\left(X_{i}>x, X_{j}>a(x) z\right) & =\int_{x}^{\infty} \int_{a(x) z}^{\infty} \eta_{i j}(s, t) F_{i}(\mathrm{~d} s) F_{j}(\mathrm{~d} t) \\
& \leq M \bar{F}_{i}(x) \bar{F}_{j}(a(x) z) .
\end{aligned}
$$


Consequently by $(5.6)$

$$
\lim _{x \rightarrow \infty} \frac{\mathbb{P}\left(X_{i}>x, X_{j}>a(x) z\right)}{\mathbb{P}\left(X_{0}>x\right)}=0
$$

and thus the proof follows by applying Corollary 3.2 of Mitra and Resnick (2009).

Proof of Theorem 5.3.: Since $\Upsilon_{i}, 1 \leq i \leq n$ are upper bounded and $F$ is a subexponential distribution, then (5.8) implies

$$
\psi(u ; n) \sim \sum_{k=1}^{n} \mathbb{P}\left(R_{k} \prod_{i=1}^{k} \Upsilon_{i}>u\right)=\sum_{k=1}^{n} \mathbb{P}\left(R_{k}^{+} \prod_{i=1}^{k} \Upsilon_{i}>u\right), \quad u \rightarrow \infty .
$$

By the fact that $R_{1}, \ldots, R_{n}$ are independent of $\Upsilon$, then for each $1 \leq k \leq n$ the random vector $\left(R_{k}, \Upsilon_{1}, \ldots, \Upsilon_{k}\right)$ has the joint $\mathrm{df}$

$$
\eta_{\Upsilon_{\mathbf{k}}} \cdot d\left(F \prod_{i=1}^{k} G_{i}\right)
$$

where $\eta \Upsilon_{\mathbf{k}}$ is defined in (5.9). Consequently, the proof of (5.10) follows by Theorem 4.2.

Proof of Theorem 5.4.: The proof is similar to that of Theorem 5.3 by using (5.8) and Theorem 4.1 a).

Acknowledgement: We greatly thank Professor Qihe Tang for numerous discussions and suggestions as well as for sending us several interesting manuscripts related to the subject of this paper. We are in debt to the referees for significant improvements of our initial submission. Enkelejd Hashorva gratefully acknowledges the support from Swiss National Science Foundation Project 200021-134785. Research of Yang Yang has been supported by National Natural Science Foundation of China (No. 11001052, 71171046), China Postdoctoral Science Foundation (No. 2012M520964), Qing Lan Project, Project of Construction for Superior Subjects of Statistics \& Audit Science and Technology of Jiangsu Higher Education Institutions.

\section{REFERENCES}

[1] Arendarczyk, M. and Dębicki, K., 2011. Asymptotics of supremum distribution of a Gaussian process over a Weibullian time. Bernoulli, 17, 194-210.

[2] Arendarczyk, M. and Dębicki, K., 2012. Exact asymptotics of supremum of a stationary Gaussian process over a random interval. Stat. Probab. Lett., 82, 645-652.

[3] Asimit, A.V., Furman, E., Tang, Q. and Vernic, R., 2011. Asymptotics for risk capital allocations based on conditional tail expectation. Insurance Math. Econom. 49, 310-324.

[4] Asimit, A. V. and Badescu, A.L., 2010. Extremes on the discounted aggregate claims in a time dependent risk model. Scand. Actuar. J. 2, 93-104.

[5] Berman, M.S., 1992. Sojourns and Extremes of Stochastic Processes, Wadsworth \& Brooks/ Cole, Boston.

[6] Breiman, L., 1965. On some limit theorems similar to the arc-sin law. Theory Probab. Appl. 10, 323-331.

[7] Chen, Y., 2011. The finite-time ruin probability with dependent insurance and financial risks. J. Appl. Probab. 48, $1035-1048$.

[8] Chen, Y. and Yuen, K.C. 2009. Sums of pairwise quasi-asymptotically independent random variables with consistent variation. Stoch. Models 25, 76-89.

[9] Chen, Y. and Yuen, K.C. 2012. Precise large deviations of aggregate claims in a size-dependent renewal risk model. Insurance Math. Econom. 51, 457-461.

[10] Cline, D.B.H. and Samorodnitsky, G., 1994. Subexponentiality of the product of independent random variables. Stochastic Process. Appl. 49, 75-98.

[11] Constantinescu, C., Hashorva, E. and Ji, L., 2011. The Archimedean copula in finite and infinite dimensions - with applications to ruin problems. Insurance: Mathem and Econom. 49, 487-495.

[12] Davis, R.A. and Resnick, S.I., 1988. Extremes of moving averages of random variables from the domain of attraction of the double exponential distribution. Stochastic Process. Appl. 30, 41-68. 
[13] Denuit, M., Dhaene, J., Goovaerts, M. and Kass, R., 2006. Actuarial Theory for Dependent Risks: Measures, Orders and Models. Wiley.

[14] Embrechts, P., Klüppelberg, C. and Mikosch, T., 1997. Modelling Extremal Events. Springer, Berlin.

[15] Geluk, J. and Tang, Q., 2009. Asymptotic tail probabilities of sums of dependent subexponential random variables. J. Theoretical Probab. 22, 4, 871-882.

[16] Goovaerts, M., Kaas, R., Laeven, R., Tang, Q. and Vernic, R., 2005. The tail probability of discounted sums of Pareto-like losses in insurance. Scandinavian Actuarial Journal 6, 446-461.

[17] Hashorva, E. and Hüsler, J., 1999. Extreme values in FGM random sequences. J. Multiv. Analysis 68, 2, $212-225$.

[18] Hashorva E., 2013a. Exact tail asymptotics of aggregated parametrised risk. J. Math. Anal. Appl., 400, 1, $187-199$.

[19] Hashorva, E., 2013b. Minima and maxima of elliptical triangular arrays and spherical processes. Bernoulli, in press, DOI 10.3150/12-BEJ463.

[20] Hashorva, E., 2012. Exact tail asymptotics in bivariate scale mixture models. Extremes, 15, 109-128.

[21] Hashorva, E., Ji, L., Tan, Z., 2012. On the infinite sums of deflated Gaussian products. Elect. Comm. Probab., 17, $31,1-8$.

[22] Hashorva, E., 2011. On beta-product convolutions. Scandinavian Actuarial J. doi:10.1080/03461238.2011.555939.

[23] Hashorva, E. and Pakes, A.G., 2010. Tail asymptotics under beta random scaling. J. Math. Anal. Appl. 372, $496-514$.

[24] Hashorva, E., Pakes, A.G. and Tang, Q., 2010. Asymptotics of random contractions. Insurance Math. Econom. 47, $405-414$.

[25] Haug, S., Klüppelberg, C. and Peng, L., 2011. Statistical models and methods for dependence in insurance data. J. Korean Stat. Soc. 40, 125-139.

[26] Jessen, A.H. and Mikosch, T., 2006. Regularly varying functions. Institut Mathématique. Publications. Nouvelle Série 80, $171-192$.

[27] Jiang, J. and Tang, Q., 2011. The product of two dependent random variables with regularly varying or rapidly varying tails. Stat. Probab. Lett. 81, 957-961.

[28] Kortschak, D., Hashorva, E., 2013. Efficient simulation of tail probabilities for sums of log-elliptical risks. J. Comp. Appl. Math., in press.

[29] Kortschak, D., 2011 Second order tail asymptotics for the sum of dependent, tail-independent regularly varying risks. Extremes, 15, 353-388.

[30] Li, J., Tang, Q. and Wu, R., 2010. Subexponential tails of discounted aggregate claims in a time-dependent renewal risk model. Adv. in Appl. Probab. 42, 1126-1146.

[31] Liu, Y. and Tang, Q., 2010. The subexponential product convolution of two Weibull-type distributions. J. Aust. Math. Soc. 89, $277-288$.

[32] Manner, H. and Segers, J., 2011. Tails of correlation mixtures of elliptical copulas. Insurance Math. Econom. 48, 153-160.

[33] Mikosch, T., 2009. Non-Life Insurance Mathematics: An Introduction with Poisson Process, Second Edition. Springer, Berlin.

[34] Mitra, A. and Resncik, S.I., 2009. Aggregation of rapidly varying risks and asymptotic independence. Adv. Appl. Probab. 41, $797-828$.

[35] Resnick, S.I., 1987. Extreme Values, Regular Variation and Point Processes. Springer, New York.

[36] Resnick, S.I. and Willekens, E., 1991. Moving averages with random coefficients and random coefficients autoregressive models. Commun. Stat. Stoch. Models 7, 511-525.

[37] Tang, Q., 2006a. On convolution equivalence with applications. Bernoulli 12, 535-549.

[38] Tang, Q., 2006b. The subexponentiality of products revisited. Extremes 9, 231-241.

[39] Tang, Q., 2008. From light tails to heavy tails through multiplier. Extremes 11, 379-391.

[40] Tang, Q., Vernic, R. and Yuan, Z., 2011. The finite-time ruin probability in the presence of dependent extremal insurance and financial risks. Preprint.

[41] Yang, Y., Hu, S. and Wu, T., 2011. The tail probability of the product of dependent random variables from max-domains of attraction. Stat. Probab. Lett. 81, 1876-1882.

[42] Yang, Y. and Wang, Y., 2012. Tail behavior of the product of two dependent random variables with applications to risk theory. Extremes doi:10.1007/s10687-012-0153-2. 